
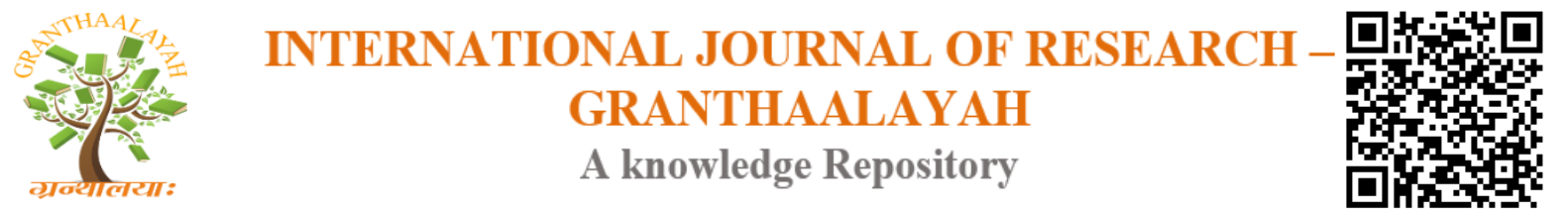

Science

\title{
WHY DOES THE STRUGGLE AROUND SRT CONTINUE TO THIS DAY?
}

\author{
A. Chubykalo ${ }^{1}$, A. Espinoza ${ }^{1}$, V. Kuligin ${ }^{2}$, M. Korneva ${ }^{2}$ \\ ${ }^{1}$ Unidad Académica de Física, Universidad Autónoma de Zacatecas, A.P. C-580, Zacatecas, \\ México \\ ${ }^{2}$ Physical Faculty Department of an Electronics, Voronezh State University, Russia
}

\begin{abstract}
The purpose of this article is not to criticize the theory of relativity, but to try to understand why, despite more than a century of dominance in physics, it is constantly criticized by physicists. In this paper, a thorough analysis of A. Einstein's theory of relativity is carried out. It relies on philosophical, physical-mathematical, logical-historical methods of investigation. It is shown that in SRT there is an error in the physical interpretation of the mathematical formalism of the Lorentz transformation (epistemological error). Therefore, the interpretation of the SRT phenomena contains logical contradictions and paradoxes. It is also shown that a consistent interpretation can be given for the Lorentz transformation within the framework of classical space-time representations. It is established that the real speed of the relative motion of inertial reference frames in $1 / \sqrt{1-v^{2} / c^{2}}$ is greater than the speed entering the Lorentz transformation. A new explanation is offered for relativistic phenomena without violating logic and without paradoxes. The results are of great importance for the description of relativistic phenomena in physical theories, and also for applied disciplines, for example, for the theory of cyclic accelerators, etc.
\end{abstract}

Keywords: Epistemological Errors; Galileo Parametric Transformation; Space; Time; Theory of Cognition.

Cite This Article: A. Chubykalo, A. Espinoza, V. Kuligin, and M. Korneva. (2019). "WHY DOES THE STRUGGLE AROUND SRT CONTINUE TO THIS DAY?." International Journal of Research - Granthaalayah, 7(1), 205-237. 10.29121/granthaalayah.v7.i1.2019.1050.

\section{Introduction}

The idea of this article was born after careful reading of two recently published works [1] and [2]. The struggle around SRT does not stop until now. Moreover, neither side can recognize itself as defeated. In this article we are not going to criticize the fundamentals of the theory of relativity, we just want to understand why, despite the more than a century domination of this theory in physics, it will continue to be subjected to serious criticism from many physicists. More than 100 years have passed of the undivided dominance of the Special theory of relativity in physics. However, the paradoxes of this theory do not have gotten convincing explanations, and the debate about the correctness of SRT continues to this day (see, for example, numerous references in Refs. 
1 and 2). We will not consider hypotheses that "destroy" the paradoxes of SRT. We found our original way of analysis.

Although scientists are tired of discussing the paradox of twins, we cannot avoid this discussion. It is important for us to understand the cause of the paradoxes by the example of this particular paradox. So let the two twin brothers live. They live in different parts of the city. Once they contacted and decided to visit each other. They did not hesitate, simultaneously got into buses and went on a visit to each other. In the middle of the road, buses rushed past each other. Brother-1 saw his brother out of the window and exclaimed: "What a young brother I have! I'm bald, and he has a young bang!". At the same time, Brother-2 also saw his moving brother in the window and exclaimed: "My brother 1 has not grown old at all! He has the red curls of a young man!"

On the one hand, we have simultaneously two mutually exclusive statements:

1) Brother-2 is younger than Brother-1 (Brother-1's affirmation).

2) Brother-1 is younger than Brother-2 (Brother-2's affirmation).

On the one hand, the assertions contain a logical contradiction, the opponents of SRT rightly assert. The scientific theory can not contain logical contradictions. Opponents of SRT are right.

On the other hand, in 1906, Poincaré expressed an assertion about the equality of inertial frames of reference and the unity of laws in such frames of reference [3]. This is a fundamental position that corresponds to a materialistic worldview.

The refusal of the Lorentz transformation is simultaneously a rejection of the equality of reference systems. This means the existence of the "navel of the universe"; distinguished, absolute reference frame. In every system that moves uniformly with respect to this absolute system, there will be physical laws that differ from the laws of the absolute frame of reference.

So, we have a dialectical contradiction that needs to be resolved. Modern physicists have reduced the role of philosophy to methodology and do not know how to conduct an epistemological analysis of physical theories. Physicists have lost faith in modern philosophy. The skeptical attitude of physicists towards philosophy is a clear proof of this fact.

However, physics cannot develop outside the philosophical worldview. Any interpretation of phenomena is a reflection of the logic of qualitative relations and relations, is a reflection of the worldview positions of the researcher. Philosophical "vacuum" formed in the early 20th century, physics must overcome themselves. For this reason, we will begin by analyzing certain philosophical problems. We will not consider the content of the categories "phenomenon and essence". The reader will find detailed information about philosophical categories in any philosophical textbook. We consider the features that allow us to separate these categories. This is the first necessary step.

\section{Observer, Phenomenon, Essence}

"The essence is known through the phenomenon." This old philosophical rule we must give concrete content. We will outline this problem in a popular form. So, there is a knowing subject, 
which in SRT we call the "observer". There is an object of observation, for example, a planet or other light source. The information about the observed object should transfer some "information carrier" to the subject. This information is a "phenomenon".

Typically, the information carrier is light, but information can carry sound waves, etc. When distributed, information may be distorted due to propagation conditions. These "distortions" engineers and scientists widely use. Examples include microscopes, telescopes, magnifiers, etc. A subject, observing the phenomena, tries to understand their essence.

\section{1. "Golden Rule"}

How to distinguish between the phenomenon and essence? We will not turn to philosophical encyclopedias. We will try to formulate a simple rule, convenient for use by physicists.

Let us start with a simple example. You received a beautiful diamond. You keep this diamond in your hands. You turn it look through the magnifying glass. All that you observe is a set of phenomena. Each phenomenon is unique and reflects the specific characteristics of the cut. However, you are interested in the essence. A set of phenomena forms the researcher a certain image of the object under study. Essence is the content of the results of observation, which does not depend on the conditions of observation. To the characteristics of the essence can be attributed, for example, cutting a diamond. It does not depend on the conditions of observation. There are other, no less important for you, characteristics of the essence. For example, you need to understand: do you deal with a natural mineral or is it a talented fake? Alternatively, for example, you are interested in the question: are there any cracks inside the diamond, air bubbles, and foreign inclusions? You put the diamond in your pocket. Phenomena have disappeared, but the diamond itself and its essence have remained! They rest in your pocket! So, we can formulate a "golden rule":

The phenomenon depends on the conditions of observation, and on the contrary, the essence does not depend on the conditions of observation.

We give one more illustration (Figure 1). It depicts the cylinder and the projection of the cylinder on the orthogonal planes. The cylinder is a kind of essence. Projections of a cylinder on a plane are phenomena that the observer (or observers) are studying (measuring). These projections depend on the condition, i.e. from the orientation of the $O O^{\prime}$-axis of the cylinder relative to the planes. We can change the condition to study a set of phenomena.

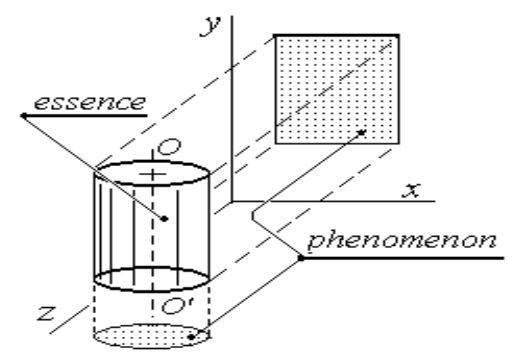

Figure 1: Illustration of philosophical categories of essence and phenomenon on the example of projections of a cylinder on orthogonal planes. 
It is impossible to establish an essence from one phenomenon! Using only one projection on the plane, we cannot describe the three-dimensional structure of the object. In addition, the observer can not other sides of the essence (the internal structure of the cylinder, material, etc.). Therefore, philosophers talk about the essences of the first, second and other orders.

Now, having in our hands a "golden rule", we can begin to analyze the linear paradoxes of the theory of relativity of A. Einstein. Already now we can say that in relativistic theories there is no such division into phenomenon and essence. Everything that the observer fixes is actually existing without distortion, essence. The twin observer fixes in the consciousness the slower pace of life of his moving twin brother and concludes that the brother is "younger" and there is a "slowing down of time" in the moving frame of reference, etc. Below we will consider these issues.

\subsection{Errors in Physics of the Early 20 Century}

One should not think that A. Einstein is the founder of the above error. Confusion with philosophical categories "phenomenon - essence" existed long before Einstein. For example, such a mistake was made by Einstein's "idol" scientist E. Mach. Now we will show the "stump", about which physicist Mach and philosopher Avenarius stumbled. V. I. Lenin in the book "Materialism and Empirio-Criticism" severely criticizes their philosophical conclusions. We want to draw attention to the starting point, which initiated the error of Mach. We quote Lenin's Materialism and Empirio-Criticism [4]:

“...We saw that Marx in 1845, Engels in 1888 and 1892, Introduce the criterion of practice as the basis of the theory of cognition of materialism. Outside of the practice of raising the question of whether "the objective" (i.e., objective) "truth" corresponds to human thinking, there is scholasticism, "Marx says in his second thesis on Feuerbach. The best refutation of Kantian and Humean agnosticism, as well as of other philosophical quirks (Schrullen), is practice, Engels repeats. "The success of our actions proves the agreement (conformity, Übereinstimmung) of our perceptions with the objective (objective) nature of perceived things," Engels objects to the agnostics." Compare this with Mach's reasoning about the criterion of practice. "In everyday thinking and everyday speech, one usually opposes the seeming, illusory reality. Holding a pencil in front of us in the air, we see him in a straight position; having lowered it in an inclined position in water, we see it bent. In the latter case they say: "The pencil seems bent, but in reality it is straight." But on what basis do we call one fact a reality, and the other we reduce to the meaning of an illusion?... When we make that natural mistake, that in cases of extraordinary we are still waiting for the onset of ordinary phenomena, our expectations, of course, are deceived. But the facts are not to blame. To speak in such cases of illusions makes sense from the practical point of view, but not at all scientific. In the same way, it makes no sense from the point of view of the scientific question often discussed, is there really a world, or is it just our illusion, nothing more than a dream. But even the most incongruous dream is a fact, no worse than any other." ("Analysis of sensations", pp. 18-19)." (Bold selection - Auth.)

Now the word to us. We are considering a "pencil", and the pencil we see is a phenomenon. Looking at the end, we see a hexagon, and looking from the side, we see a rectangle. If we lower the end of the pencil obliquely into a glass of water, we will see it "broken". All these are phenomena behind which the essence is hidden from Mach. Mach became entangled, not knowing 
the criteria for distinguishing the phenomenon from the essence and, as a result, fell into idealism. Lenin writes in the same place [4]:

"This is precisely such a tortured professorial idealism, when the criterion of practice, which separates illusion from reality for everyone, is carried out by E. Mach beyond science, beyond the theory of cognition."

Now we can proceed to the analysis of linear paradoxes of SRT.

\section{Paradoxes of SRT in the Context of the Theory of Cognition}

We will note the following. Light rays deliver information to the observer. It is not accidental that in all mental experiments, Einstein used light rays. Light rays, as we know, can deliver distorted information.

\subsection{Example of Analysis of Paradoxes}

We propose it as an illustration of the structure of the linear paradoxes of SRT. So, let two identical bars, standing vertically, be separated by a concave lens, as shown in Figure 2. The first observer considers the construction on the left, the second observer looks at the construction on the right. The left observer sees in front of him a black rod $H_{b}$ and through the lens he sees a white rod $h_{w}$. It seems to the left observer that the black rod is longer than the white one $H_{b}>h_{w}$. The right observer claims the opposite. He thinks the white rod is longer than the black rod, $H_{w}>h_{b}$. Which of the bars is higher in reality?

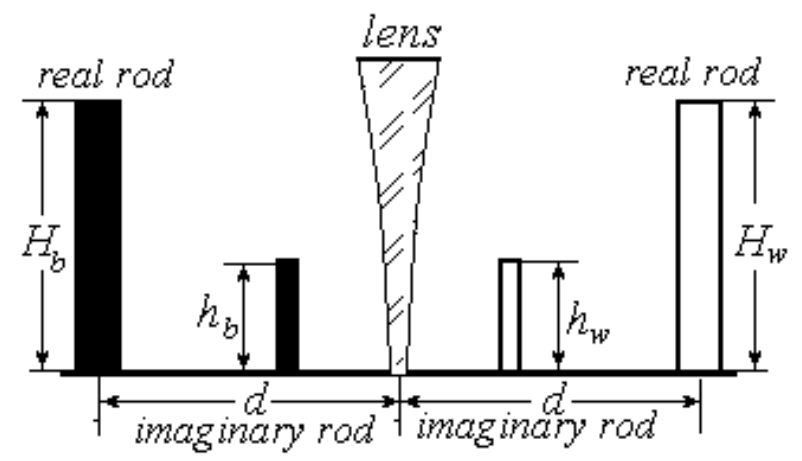

Figure 2: Illustration for example about the essence and phenomenon

We give an obvious answer. We make a gross mistake if we identify the characteristic of a phenomenon with the characteristic of "essence". We have no right to consider the height of the imaginary image $h_{b}$ or $h_{w}$ as an essence. The values of $h_{b}$ or $h_{w}$ depend on the distance $d$. The distance $\mathrm{d}$ is the observation condition (the "golden rule"). Consequently, $h_{b}$ and $h_{w}$ are the characteristics of the phenomenon, i.e. they are a distorted mapping of the essence. The values of $H_{b}$ and $H_{w}$ do not depend on the condition, i.e. from the distance to the lens $d$. They are characteristics of the essence. Thus, the contradiction is easily eliminated. We will note one more important aspect. The change in the observed height of the rod is due to the distortion of the front of the light wave. 
Let us pass to the paradoxes of SRT, using the "golden rule". Recall that the condition in SRT is the relative motion velocity $v$. The characteristics that do not depend on the velocity $v$ are the characteristics of the essence. If the characteristic depends on the relative velocity $v$, then it is a characteristic of the phenomenon.

\section{2. "Compression of Scale (Or Shortening of Length)"}

Let two observers have the same ruler. The length of the ruler of each observer (twin) is $l_{0}$. When observers fly past each other, they compare the lengths of the rulers.

Observer-1 claims that his ruler $l_{0}$ is longer than observer-2's ruler $l_{2}$ :

$$
l_{2}<l_{0}, l_{2}=l_{0} \sqrt{1-\frac{v^{2}}{c^{2}}} .
$$

Observer-2 claims that his ruler $l_{0}$ is longer than observer-1's ruler $l_{1}$ :

$$
l_{1}<l_{0}, l_{1}=l_{0} \sqrt{1-\frac{v^{2}}{c^{2}}} .
$$

We see that $l_{1}$ and $l_{2}$ depend on the velocity $v$. Consequently, $l_{1}$ and $l_{2}$ are characteristics of phenomena ("golden rule"). These characteristics differ from the real length $l_{0}$ (characteristic of the essence). The reason is the same as in Example 1 (Fig. 2). The front of the wave of the light beam has different directions in different inertial frames of reference. Therefore, distortion of the wave front leads to an apparent shortening of the length of the moving ruler. A similar distortion of the front is used in optical instruments.

We conclude: the real space does not depend on the inertial frame of reference, and distortions are caused by a change in the direction of the front of the light wave. The space is common to all systems.

\section{3. "Slowdown (Or Dilation) of Time"}

We will change the thought experiment of Einstein a little. Let both twins have "LEDs" with green light. The period of oscillations is equal to $T_{0}$. As in the previous example, the brothers move with a relative speed $v$. When the brothers meet, they compare the periods of observed oscillations. Fixed brother- 1 sees the yellow glow of the LED of the brother- 2 moving past him and the green glow of his LED. The observed oscillation period $T_{2}$ is larger than the period of oscillation $T_{0}$ of the stationary LED:

$$
T_{2}>T_{0}, T_{2}=\frac{T_{0}}{\sqrt{1-\frac{v^{2}}{c^{2}}}} .
$$


Moving brother-2 rests in his frame of reference. He sees the yellow light coming from the diode of the flying brother- 1 . The oscillation period $T_{1}$ observed by the brother- 2 is greater than the period of oscillation $T_{0}$ of the fixed brother-2 LED:

$$
T_{1}>T_{0}, \quad T_{1}=\frac{T_{0}}{\sqrt{1-\frac{v^{2}}{c^{2}}}} .
$$

We use the "golden rule". The periods $T_{1}$ and $T_{2}$ depend on the velocity of the relative motion $v$. Consequently, the periods $T_{1}$ and $T_{2}$ are phenomena. The periods $T_{1}$ and $T_{2}$ are the distorted projections of the entity $T_{0}$ into the moving observer's reference frame. This phenomenon is called the "transverse Doppler effect". The real time does not depend on the choice of the inertial frame of reference. It is the same for all inertial systems.

\subsection{Preliminary Conclusions}

1) A. Einstein mathematically correctly derived formulas (3.1) and (3.2). However, he gave an incorrect explanation of the results obtained (misunderstanding of philosophy). As Engels wrote, philosophy, as a whimsical lady, avenges natural science in retrospect, for the fact that the latter left it.

2) The space for all inertial systems is common. There are no real "compression of scales".

3) The time is the same for all inertial frames. There is no real "slowing down of time" in nature.

So, we have returned to the classical space-time relations. This is natural.

Now we are faced with the task of giving a new explanation to the Lorentz transformation. It turns out that there exists a parametric Galilean transformation. This transformation also preserves the speed of light unchanged in inertial frames of reference. We will first be acquainted with this transformation.

\section{Parametric Galilean Transformation}

\subsection{Parametric Transformation}

Physicists "have not discovered" to date that there is a parametric Galilean transformation. In mathematics, a transformation describes the displacement along one coordinate axis. For example, $x^{\prime}=x-a$. Here the number $a$ is the displacement parameter of the $x^{\prime}$-axis with respect to the $x$ axis. Three other independent variables of two inertial frames of reference are rigidly related by identity:

$$
y=y^{\prime} ; z=z^{\prime} ; \quad t=t^{\prime}
$$

The variables $y, z$ and $t$ do not depend on the transformation $x^{\prime}=x-a$. Nothing will change if the parameter $a$ depends on $t: x^{\prime}=x-a(t)$. In the special case $x^{\prime}=x-V t$. 
So, with the new approach, we take into account the oneness of time in the reference frames being compared and also the invariance of the $y$ and $z$ coordinates. As already mentioned, the coordinates $y, z$ and time $t$ in two frames of reference are always the same.

In the primed reference system, the wave equation has the form (the particle is at rest):

$$
\frac{\partial U^{2}}{\partial x^{\prime 2}}+\frac{\partial U^{2}}{\partial y^{2}}+\frac{\partial U^{2}}{\partial z^{2}}-\frac{1}{c^{2}} \frac{\partial U^{2}}{\partial t^{2}}=q \delta\left(\mathbf{R}_{0}^{\prime}, t\right) .
$$

The partial derivatives of the potential $U$ with respect to $x^{\prime}$ can be calculated quite simply:

$$
\frac{\partial U}{\partial x^{\prime}}=\frac{\partial U}{\partial x} \frac{\partial x}{\partial x^{\prime}}=\frac{\partial U}{\partial x} ; \quad \frac{\partial U^{2}}{\partial x^{\prime 2}}=\frac{\partial U^{2}}{\partial x^{2}} .
$$

Thus, the expression (4.2) in the new inertial system takes the final form:

$$
\frac{\partial U^{2}}{\partial x^{2}}+\frac{\partial U^{2}}{\partial y^{2}}+\frac{\partial U^{2}}{\partial z^{2}}-\frac{1}{c^{2}} \frac{\partial U^{2}}{\partial t^{2}}=q \delta(\mathbf{R}-\mathbf{V} t, t)
$$

We repeat that it is not necessary to transform the equation by other coordinates and time.

Here are the preliminary conclusions:

- Space is common to all inertial systems, and time is one for them.

- All inertial systems are equal, the speed of light $\mathrm{c}$ does not depend on the choice of the inertial reference frame by the observer (Poincaré, 1906 [3]).

\subsection{Phenomena with Relative Motion}

Now we need to write down the equations to state the effects.

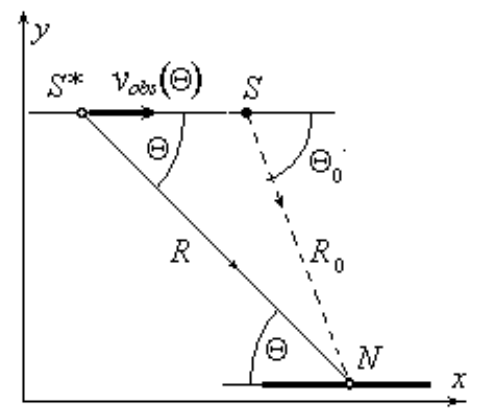

Figure 3

In Fig. 3 shows the classical phenomenon of aberration in the observer's reference frame. We write the equations.

$$
\mathbf{R}=\mathbf{R}_{0}+\mathbf{V T} ; \quad T=R_{0} / c
$$


Equations are obtained the same in the reference system of the radiation source and in the reference frame of the observer (the equivalence of inertial systems). We will write the equations in expanded form for the observer's reference frame (See Figure 3):

$$
R \cos \Theta=R_{0} \cos \Theta_{0}+V T ; \quad R \sin \Theta=R_{0} \sin \Theta_{0} ; \quad T=R_{0} / c .
$$

From (4.2.2) the following relations for angles follow:

$$
\begin{aligned}
& \sin \Theta=\frac{\sin \Theta_{0}}{\sqrt{1+2 \frac{V}{c} \cos \Theta_{0}+\left(\frac{V}{c}\right)^{2}}} ; \\
& \cos \Theta=\frac{\cos \Theta_{0}+V / c}{\sqrt{1+2 \frac{V}{c} \cos \Theta_{0}+\left(\frac{V}{c}\right)^{2}}} ; \\
& \sin \Theta_{0}=\left[\frac{V}{c} \cos \Theta+\sqrt{1-\left(\frac{V}{c} \sin \Theta\right)^{2}}\right] \sin \Theta \\
& \cos \Theta_{0}=-\frac{V}{c} \sin ^{2} \Theta+\cos \Theta \sqrt{1-\left(\frac{V}{c} \sin \Theta\right)^{2}} .
\end{aligned}
$$

The expressions (4.2.5) and (4.2.6) are limited by the inequality $\frac{V}{c}|\sin \Theta| \leq 1$.

Distortion factor of distance- We introduce the distortion coefficient $n_{g}$, which connects the apparent and real distances

$$
n_{g}=\frac{R}{R_{0}}=\frac{\sin \Theta}{\sin \Theta_{0}}=\frac{V}{c} \cos \Theta+\sqrt{1-\left(\frac{V}{c} \sin \Theta\right)^{2}} .
$$

At relative velocities $V$, which are much smaller than the speed of light $c$, we have the following approximate expression:

$$
n_{g} \approx 1+\frac{V}{c} \cos \Theta
$$

The observed velocity- The expression for the observed velocity is derived in the standard way:

$$
v_{\mathrm{obs}}=\frac{V}{1-\frac{V}{c} \cos \Theta} \text {. }
$$


The Doppler effect- The Doppler effect is described by a similar formula:

$$
f_{\text {obs }}=\frac{f_{0}}{1-\frac{V}{c} \cos \Theta},
$$

where $f_{0}$ is the oscillation frequency in the reference frame of the source.

Angle of aberration- This angle of aberration for rectilinear motion is $\delta=\Theta_{0}-\Theta$. It is easy to calculate, based on geometric considerations (see Figure 3)

$$
\sin \delta=\sin \left(\Theta_{0}-\Theta\right)=\frac{V}{c} \sin \Theta .
$$

\subsection{Rotating Frame of Reference}

Inertial frame of reference- Let us assume that the light source $S$ moves around the observer $N$ along a circular orbit with angular velocity $\Omega\left(\varphi^{\prime}=\Omega t\right)$. The observer is at rest in the center of this orbit. The angular velocity $\Omega$ of the light source $S$ is constant.

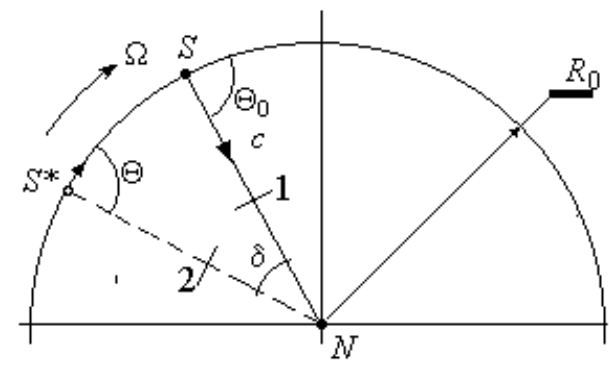

Figure 4: Circular motion of a light source. 1 - undistorted wave front; 2 - distorted wave front.

Let the light source $S$ emit a light pulse to the observer at time $t=t_{\text {rad }}=0$. The observer $N$ will accept this pulse at the instant $t=T=R_{0} / c$. During the time $T$, the moving source moves to position $S$. (See Fig. 4). The angle of aberration is $\delta=R_{0} \Omega / c$. The light pulse moves along a straight line $S^{*} N$ at the speed of light. The angle $\varphi^{\prime}$ for the light pulse is constant. The equation of motion of the light pulse from the source $\mathrm{S}$ to the observer has the form:

$$
r=R_{0}-c t ; \quad \varphi^{\prime}=0,
$$

where $r$ is the present coordinate of the light pulse.

Noninertial reference frame- We now consider the same phenomena in a noninertial system in which the source $\mathrm{S}$ is at rest. We write the wave equation in a cylindrical coordinate system for an inertial frame of reference:

$$
\frac{1}{r} \frac{\partial}{\partial r}\left(r \frac{\partial u}{\partial r}\right)+\frac{1}{r^{2}} \frac{\partial u^{2}}{\partial \varphi^{2}}+\frac{\partial u^{2}}{\partial z^{2}}-\frac{1}{c^{2}} \frac{\partial u^{2}}{\partial t^{2}}=0
$$


It turns out that for equation (4.3.1) there exists a transformation analogous to the linear parametric Galilean transformation $\left(x=x^{\prime}-v t\right)$. This transformation preserves equation (4.3.1) unchanged. The transformation is:

$$
r=r^{\prime} ; \quad \varphi=\varphi^{\prime}-\Omega t ; \quad z=z^{\prime} ; \quad \Theta=\Theta^{\prime}
$$

The quantities $t, r$, and $z$ are independent on $\varphi=\varphi^{\prime}-\Omega t$. The parametric transformation preserves the form of the wave equation in the rotating frame of reference. In the new frame of reference, the observer rotates counterclockwise at an angular velocity $\Omega$ around its axis. The light source $S$ is at rest. We now write the equations describing the motion of the light pulse from the source $S$ to the observer $N$. In the inertial system, the trajectory of the pulse is a fixed straight line along the radius. The angle $\varphi^{\prime}$ was constant. In a noninertial reference system, a straight line (trajectory) will rotate at an angular velocity counterclockwise. Simultaneously, the light pulse will move to the axis of rotation.

$$
r=R_{0}-c t ; \quad \varphi=-\Omega t
$$

You can enter the length of the arc instead of the angle:

$$
s=r \varphi^{\prime}=-r \Omega t
$$

The resulting parametric equations (4.3.3) and (4.3.4) describe the trajectory of the light pulse as it moves from $S$ to $N$ in a rotating frame of reference.

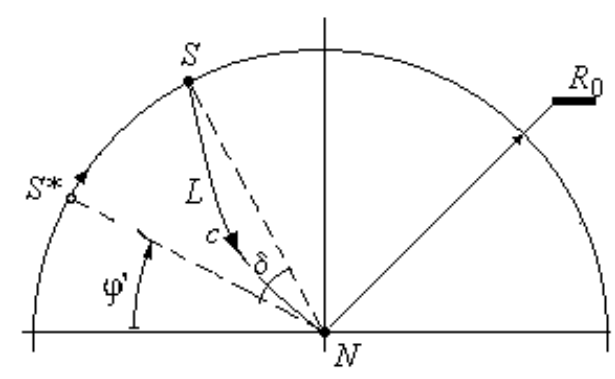

Figure 5: Noninertial reference frame.

Let the light pulse be emitted at $t=0$. It is easy to see that at the moment $T=R_{0} / c$ the observer receives this pulse, the aberration angle is equal to $\delta=R_{0} \Omega / c$. This result corresponds to the result obtained earlier for an inertial frame of reference. We also note that the trajectory of the light ray $L$ in the rotating frame of reference became longer (phenomenon). Therefore, the ratio of the circumference to the diameter is less than $2 \pi\left(2 \pi R_{0} / L \leq 2 \pi\right)$.

So, we have considered kinematic phenomena using Galileo's parametric transformation. The Galilean transformation has the following properties:

1) The transformation uses classical space-time relations.

2) The speed of light is constant in any inertial frame of reference.

3) The transformation has a simple mathematical apparatus.

4) The transformation has a clear physical interpretation. 
We can consider the parametric transformation of Galileo as an alternative to the Lorentz transformation. The choice will help make experimental studies. For example, the transverse Doppler effect in the parametric Galilean transformation is absent. We note that the results of the Galileo transformation coincide with the results of the Lorentz transformation, at low velocities up to terms $v^{2} / c^{2}$.

Now we need to give a new interpretation to the phenomena within the framework of the Lorentz transformation. We recall that this interpretation is based on classical space-time relations and should not lead to paradoxes and contradictions. Moreover, the new interpretation should not use special hypotheses and postulates.

\section{The Lorentz Transformation}

Preliminary comment- In the previous chapter, we explained the main cause of the paradoxes of SRT by the epistemological error admitted by A. Einstein. To give the Lorentz transformation a correct interpretation, we must return to the classical concepts of space and time. Euclidean space is common to all inertial frames of reference. The space is homogeneous and isotropic. Time is the same for inertial systems. Time is uniform and continuous. All inertial systems are equal.

It is interesting to note that the Lorentz transformation is not the only transformation that keeps Maxwell's equations unchanged (Appendix 1). Another mistake is the use of the group properties of the Lorentz transformation. As shown in Appendix 2, the group of Lorentz-type transformations is not commutative. The absence of commutativity directly contradicts the properties of Euclidean space. We use a different path.

The textbooks state that the Lorentz transformation contradicts the transformation of Galileo. This is an old error. The Lorentz transformation depends on the relative velocity between the observer and the light source. The relative velocity is an invariant of the Galileo transformation. Consequently, the Lorentz transformation is invariant under the Galileo transformation. This allows us to find a simple way to explain and describe relativistic phenomena.

\subsection{He Phenomenon of Aberration}

Let us consider the phenomenon of aberration in the reference system of a light object (Figure 6). The observer moves with a speed $\mathbf{V}$ from the position $N^{*}$ to the point of meeting with the light beam at the point $N$. The light beam passes the distance to the meeting point $T_{0}=R_{0} / c$. The front of the light wave is perpendicular to the distance $\mathbf{R}_{0}$. The moving observer perceives this front with distortions. In the mind, the observer constructs the continuation of the ray perpendicular to the observed front. It seems to him that the wave propagates along $R$ : 


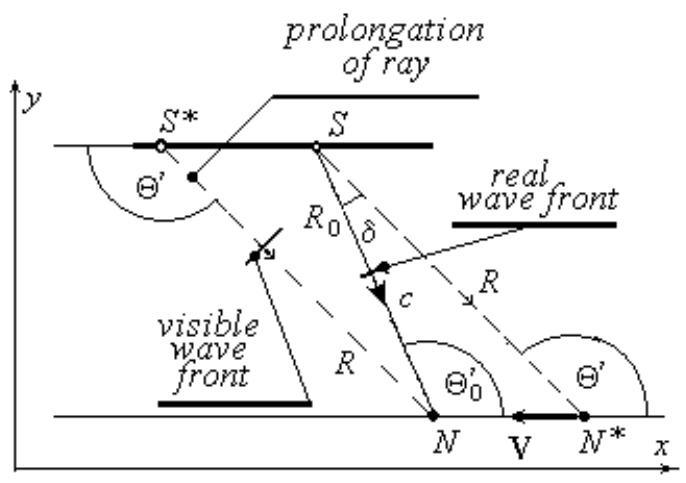

Figure 6

He sees a luminous object at the point $S^{*}$, although the real object is at the point $S$. The distance $R$ depends on the speed of relative motion. The higher the velocity $\mathbf{V}$, the greater the apparent distance $R$. Thus, we are dealing with two objects. The first object $\mathrm{S}$ is real. The second object $S^{*}$ is an imaginary representation of the real object. The difference in viewing angles $\Theta^{\prime}-\Theta_{0}^{\prime}=\delta$ is the aberration angle.

Similar phenomena occur in the observer's reference frame, as shown in Fig. 7. We will give an explanation so that we can compare the two figures. When moving from the reference frame of the light source to the observer's reference frame, the directions of vectors and angles change:

$$
\mathbf{V} \Rightarrow-\mathbf{V} ; \quad \Theta_{0}^{\prime} \Rightarrow \pi-\Theta_{0} ; \Theta^{\prime} \Rightarrow \pi-\Theta
$$

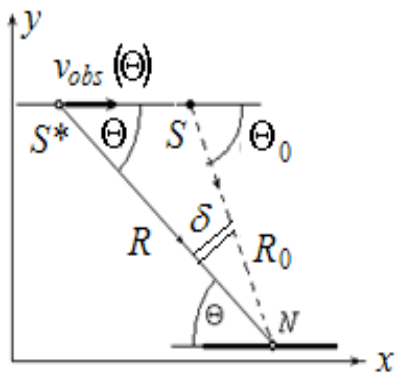

Figure 7

We see in Fig. 7 the two objects $S^{*}$ and $S$. The object $S^{*}$ is seen on the celestial sphere at the moment when the light pulse meets the observer $N$. During the propagation time of the signal $T_{0}=R_{0} / c$ the source moves to the point $S$. The angle between the vectors $\mathbf{R}$ and $\mathbf{R}_{0}$ is the angle aberrations. Since the Lorentz transformation is invariant under the Galileo transformation, the qualitative picture of the aberration phenomenon is the same for these transformations. But the quantitative relationship will be different.

Comment- To avoid mistakes in reasoning, the researcher must observe the following rule. The distance between the real radiating object and the observer is always measured when the observer receives a light pulse. 


\subsection{Observed Object Speed}

As we said above, the Lorentz transformation is invariant under the Galileo transformation. Note that in the Lorentz transformation, the true scalars are characteristics of the essence. We are now on the new side to explain the essence of the Lorentz transformation.

Let the light source $S$ move along a straight line past the resting observer $N$. The observer is provided with a clock and a ruler (Figure 7 ). The observer measures the velocity $v_{\text {obs }}$ of a visible object $S$, depending on the viewing angle $\Theta$.

We write the formula for the dependence of the observed velocity on the viewing angle:

$$
v_{\mathrm{obs}}=\frac{v}{1-\frac{v}{c} \cos \Theta} .
$$

The expression (5.2.1) is universal. It was obtained directly in the observer's reference frame. The need to use transformations arises only when two inertial systems are compared. If the real relative velocity of inertial systems is constant, we can separate the essence from the observed phenomenon, using the viewing angle $\Theta$ as a condition. All values of $v_{\text {obs }}$ are observable phenomena. They depend on $\Theta$.

When we performed the analysis within the framework of the Galileo parametric transformation in Chapter 4, the velocity $v$ in expression (5.2.1) was the real speed of the relative motion of the inertial reference frames. We measured this speed at $\Theta=90^{\circ}$. Is the speed $v$ now the real speed of relative motion in the Lorentz transformation? This question we will answer later. Now we will assume that the velocity $v$ is the observed velocity $v_{\text {obs }}$ of the object at the viewing angle $\Theta=90^{\circ}$.

\subsection{Observed and Real Values}

Previously, we wrote the universal expression (5.2.1) for the observed velocity. The same universal formulas are for the observed lengths of moving segments $\Delta x_{\mathrm{obs}}$, for the observed time intervals $\Delta t_{\mathrm{obs}}$, for the Doppler effect $f_{\mathrm{obs}}$ :

$$
\begin{gathered}
\Delta x_{\mathrm{obs}}(\Theta)=\frac{\Delta x}{1-\frac{v}{c} \cos \Theta} ; \\
f_{\mathrm{obs}}(\Theta)=\frac{f}{1-\frac{v}{c} \cos \Theta} ; \\
\Delta t_{\mathrm{obs}}(\Theta)=\Delta t\left[1-\frac{v}{c} \cos \Theta\right] .
\end{gathered}
$$

The values, $f$ and we measure at an observation angle of $90^{\circ}$. Are the values of $\Delta x, f$ and $\Delta t$ the characteristics of the essence, i.e. real values measured at $v=0$ ? If we used the parametric 
Galilean transformation, in which time does not appear in the transformation formulas, then we had a simple solution. Values of $\Delta x, f$ and $\Delta t$ are characteristics of the essence.

However, in the Lorentz transformation, the time $t$ is transformed simultaneously with the coordinate $x$. We do not have confidence that the quantities $\Delta x, f$ and $\Delta t$, are the characteristics of the essence. If they were characteristics of the essence, then we had the same results as in the Galilean transformation.

Here we need the numerical results of Einstein's "mental experiments": "compression of scale" and "time dilation". Einstein argued for the case when the subject and the object of observation were against each other. This position corresponds to the viewing angle $\Theta=90^{\circ}$.

Comparing (5.3.1) and (5.3.3) with the results of a mental experiment, we obtain:

$$
\Delta x_{\mathrm{obs}}\left(90^{\circ}\right)=\Delta x_{0} \sqrt{1-\frac{v^{2}}{c^{2}}} ; \Delta t_{\mathrm{obs}}\left(90^{\circ}\right)=\frac{\Delta t_{0}}{\sqrt{1-\frac{v^{2}}{c^{2}}}} .
$$

The index " 0 " we assign to the values measured in the reference frame of the fixed light source. Taking into account the expressions obtained, we write down the formulas (5.3.1) -(5.3.3) $\operatorname{as} \Delta x_{\text {obs }}(\Theta)=\frac{\Delta x_{0} \sqrt{1-\frac{v^{2}}{c^{2}}}}{1-\frac{v}{c} \cos \Theta} ; \quad f_{\text {obs }}(\Theta)=\frac{f_{0} \sqrt{1-\frac{v^{2}}{c^{2}}}}{1-\frac{v}{c} \cos \Theta} ; \quad \Delta t_{\text {obs }}(\Theta)=\Delta t_{0} \frac{1-\frac{v}{c} \cos \Theta}{\sqrt{1-\frac{v^{2}}{c^{2}}}}$.

The dependence of the observed velocity on the viewing angle and the actual value of the relative motion velocity will be obtained below.

\subsection{Real Speed of Relative Motion}

In the previous section, we obtained expressions for various effects within the framework of the Lorentz transformation. We have obtained expression (5.3.1) for the observed rate of relative motion of two inertial reference frames. We obtained expressions for the observed time intervals and the observed lengths of the segments (5.3.4). Now we have to determine the real speed of the relative motion of inertial systems and answer other questions. We need a special key to open this scientific secret. This key is the critical angle of observation $\Theta_{\mathrm{cr}}$.

$$
\Theta_{\mathrm{cr}}=\arccos \frac{1-\sqrt{1-\frac{v^{2}}{c^{2}}}}{v / c}
$$

The critical angle is of particular interest. The descriptions of the effects when $\Theta=\Theta_{\text {cr }}$ they are very simple. Write out the expressions for the coordinate segments and the time interval. Please note that at a critical viewing angle, the spatial and temporal segments are not distorted!

$$
\Delta x=\Delta x_{0} ; \Delta y=\Delta y_{0} ; \Delta z=\Delta z_{0} ; \Delta t=\Delta t_{0} .
$$


So, we fix the following:

- First, at a critical viewing angle, there is no distortion when displaying the time intervals and the lengths of the segments.

- Secondly, the existence of a critical angle makes it possible to always rigidly synchronize the clock of two inertial systems. For us it is not important, because the time for all inertial systems is the same. But for the apologists of the SRT this is important!

Real relative speed- Now we can determine the real speed of the relative motion of the inertial reference frames. At a critical angle, the length and time segments in the two frames of reference are the same, i.e. do not have distortion. The relative observable speed for two frames of reference is also the same for $\Theta=\Theta_{\mathrm{cr}}$.

$$
v_{\mathrm{obs}}\left(\Theta_{\mathrm{cr}}\right)=\frac{\Delta x_{\mathrm{obs}}\left(\Theta_{\mathrm{cr}}\right)}{\Delta t_{\mathrm{obs}}\left(\Theta_{\mathrm{cr}}\right)}=\frac{\Delta x_{0}}{\Delta t_{0}}=\frac{v}{\sqrt{1-\frac{v^{2}}{c^{2}}}}=V .
$$

Therefore, the velocity $V$ is the real relative velocity of the inertial frames of reference (essence). The velocity $V$ does not depend on the angle of observation. The velocity $V$ is a condition for the analysis of relativistic phenomena.

Distortion factor of distance- As in the Galileo parametric transformation, we can introduce the distortion coefficient $R_{0} / R=n_{d}$, which depends on the angle of observation:

$$
n_{d}=\left|\frac{\sin \Theta_{0}}{\sin \Theta}\right|=\frac{R}{R_{0}}=\frac{\sqrt{1-\frac{v^{2}}{c^{2}}}}{1-\frac{v}{c} \cos \Theta} .
$$

This coefficient will now constantly occur to us in the formulas. The ratio of the sines resembles the law of refraction of light when a light beam crosses the interface between media with different refractive indices. In contrast to the parametric Galilean transformation, the coefficient $n_{d}$ relates not only distances, but also other quantities at any relative velocities.

Observed length of the segment: $\Delta x=\Delta x_{0} n_{d}$.

Observed time interval: $\quad \Delta t=\Delta t_{0} / n_{d}$.

Doppler Effect:

$$
=f_{0} n_{d} .
$$

Therefore, we have described some relativistic phenomena described by the Lorentz transformation. We answered the question of the real speed of the relative motion of inertial frames in the framework of the Lorentz transformation. 
Remark- We will make an important remark. If the angle is critical, then the coefficient $n_{d}$ is always $1\left(n_{d}=1\right)$. The converse is also true, then the viewing angle is always critical $\left(n_{d}=1\right)$. This is important to bear in mind, for example, in the analysis of rotational motion.

The real speed of the relative motion of inertial frames is $V$ (see expression (5.4.3)). It is a characteristic of the essence. The speed $V$ can exceed the speed of light in a vacuum. The observed velocity $v$ in the Lorentz transformation (phenomenon), which we measure at an angle of $90^{\circ}$, cannot exceed the speed of light. It is here that Einstein's error and its negative consequences are clearly visible.

Illustration- The introduction of the actual velocity of relative motion makes it possible to give a new interpretation to relativistic phenomena, for example, to an increase in the meson lifetime, which "seems to be confirmed" by SRT. The distance traveled by mesons according to the SRT is

$$
R=v \frac{T_{0}}{\sqrt{1-\frac{v^{2}}{c^{2}}}}=v \tau
$$

where $\tau$ is the "lifetime" of the moving meson, associated with the "slowing down of time". Now we write expression (5.4.8) in a modified calibration:

$$
R=T_{0} \frac{v}{\sqrt{1-\frac{v^{2}}{c^{2}}}}=T_{0} V
$$

It follows from (5.4.9):

1) The meson lifetime does not depend on the choice of the inertial frame of reference.

2) The real velocity of relative motion of mesons does not depend on the viewing angle and can exceed the speed of light.

We repeat that time is the same for all inertial frames of reference. Thus, the postulate of the existence of a "limiting speed" is a delusion or prejudice.

Postulate is the usual hypothesis, turned into dogma. We rehabilitate the instantaneous action at a distance, rejected by physicists, that is implicitly contained in Maxwell's equations (see, for example, [5]). Without instantaneous action, it is impossible to solve many problems of electrodynamics, for example, the problem of electromagnetic mass and others. In [5] it is shown that the instantaneous action at a distance does not contradict the principle of causality.

\subsection{Modified Lorentz Transformation}

Let us return to the Lorentz transformation

$$
x=\frac{x_{0}-v t_{0}}{\sqrt{1-\frac{v^{2}}{c^{2}}}} ; y=y_{0} ; z=z_{0} ; c t=\frac{c t_{0}-v x_{0} / c}{\sqrt{1-\frac{v^{2}}{c^{2}}}} .
$$


Using the Lorentz transformation, Poincaré obtained the following formulas relating the angles $\Theta_{0}$ and $\Theta$ :

$$
\cos \Theta_{0}=\frac{\cos \Theta-\frac{v}{c}}{1-\frac{v}{c} \cos \Theta} ; \sin \Theta_{0}=\frac{\sin \Theta \sqrt{1-\frac{v^{2}}{c^{2}}}}{1-\frac{v}{c} \cos \Theta} .
$$

If in the Lorentz transformation we replace the velocity $v$ by the real velocity of the relative motion $V$, then we obtain the following expression for the transformation of the 4-coordinates:

$$
x=x_{0} \sqrt{1+\frac{V^{2}}{c^{2}}}-V t_{0} ; y=y_{0} ; z=z_{0} ; c t=c t_{0} \sqrt{1+\frac{V^{2}}{c^{2}}}-V x_{0} / c .
$$

This is a modified form of the Lorentz transformation, which is included in the transformation class. This transformation depends on the real speed of relative motion $V$. Now we can give a new record of the results. If we replace the velocity $v$ by the real velocity of the relative motion $V$ in the expression (5.5.1), then we obtain

$$
\cos \Theta_{0}=\frac{\sqrt{1+\frac{V^{2}}{c^{2}}} \cos \Theta-V / c}{\sqrt{1+\frac{V^{2}}{c^{2}}}-\frac{V}{c} \cos \Theta} ; \quad \sin \Theta_{0}=\frac{\sin \Theta}{\sqrt{1+\frac{V^{2}}{c^{2}}}-\frac{V}{c} \cos \Theta} .
$$

We use the same procedure for other effects.

$$
n_{d}=\frac{\sin \Theta_{0}}{\sin \Theta}=\frac{1}{\sqrt{1+\frac{V^{2}}{c^{2}}}-\frac{V}{c} \cos \Theta}:
$$

- Observed length of the segment: $\Delta x=n_{d} \Delta x_{0}$

- Observed time interval: $\Delta t=\Delta t_{0} / n_{d}$

- Doppler Effect: $\quad f=f_{0} n_{d}$

- Angle of aberration: $\quad \sin \delta=\sin \Theta \frac{\frac{V}{c}-\left(1+\frac{V^{2}}{c^{2}}-1\right) \cos \Theta}{\sqrt{1+\frac{V^{2}}{c^{2}}}-\frac{V}{c} \cos \Theta}$.

At small viewing angles, the aberration angle is:

$$
\sin \delta \approx \frac{V}{c} \sin \Theta .
$$


The angle of aberration shows the difference in the angular position of the observed object (imaginary image) and the angular position of the real object with rectilinear motion.

\subsection{Observed Form of the Object}

Let us assume that a moving object is a ruler of length $\Delta x_{0}$ oriented along the velocity vector $\mathbf{V}$. It is not difficult to see that the observed length of the ruler will depend on $V$ and $\Theta$. The apparent length of the ruler is:

$$
\Delta x=\frac{\Delta x_{0}}{\sqrt{1+\frac{V^{2}}{c^{2}}}-\frac{V}{c} \cos \Theta} .
$$

From this expression it follows that the known "shortening" of the scale at $\Theta=90^{\circ}$.

$$
\Delta x=\Delta x_{0} \sqrt{1-\frac{v^{2}}{c^{2}}}=\frac{\Delta x_{0}}{\sqrt{1+\frac{V^{2}}{c^{2}}}} .
$$

We multiply both sides of expression (5.6.1) by $\sin \Theta$ and transform the result

$$
\Delta x \sin \Theta=\Delta x_{0} \frac{\sin \Theta}{\sqrt{1+\frac{V^{2}}{c^{2}}}-\frac{V}{c} \cos \Theta}=\Delta x_{0} \sin \Theta_{0}=d .
$$

This result is useful to illustrate Fig. 8. The value of $d$ is the thickness of the light beam. Note that the thickness remains constant in any inertial frame of reference. If we take into account that the width of this ray does not depend on the choice of the inertial frame of reference, we can formulate the law of "refraction" of light when an observer moves from one inertial frame of reference to another. If the observer moves from one inertial system to another, then the light beam "turns" by an angle $\delta=\Theta_{0}-\Theta$ without changing the cross-section, as shown in Figure 8.
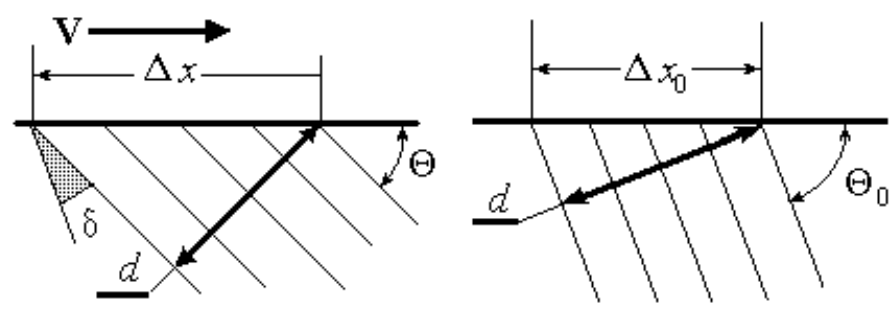

Figure 8

The result obtained allows us to describe the visible shape of a moving object. Let the cube move along a straight line. The velocity vector is perpendicular to the cube face. In its own system, the cube rests, and the rays from the cube are directed at the observer at the angle $\Theta_{0}$. The rays from the moving cube in the observer's reference frame will have a direction $\Theta$. This means that the 
observer sees the cube "turned" by the angle of aberration, as shown in Fig. 9. This is a phenomenon that we can call a visual illusion.
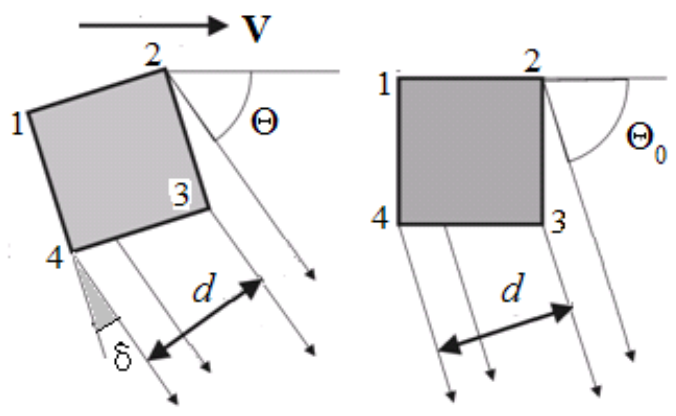

Figure 9

To complete the picture, Fig. 10 shows a series of images of a moving object (a cube of green color) perceived by the observer for several viewing angles $\theta$. The speed of the cube is $V=4 c / 3$.

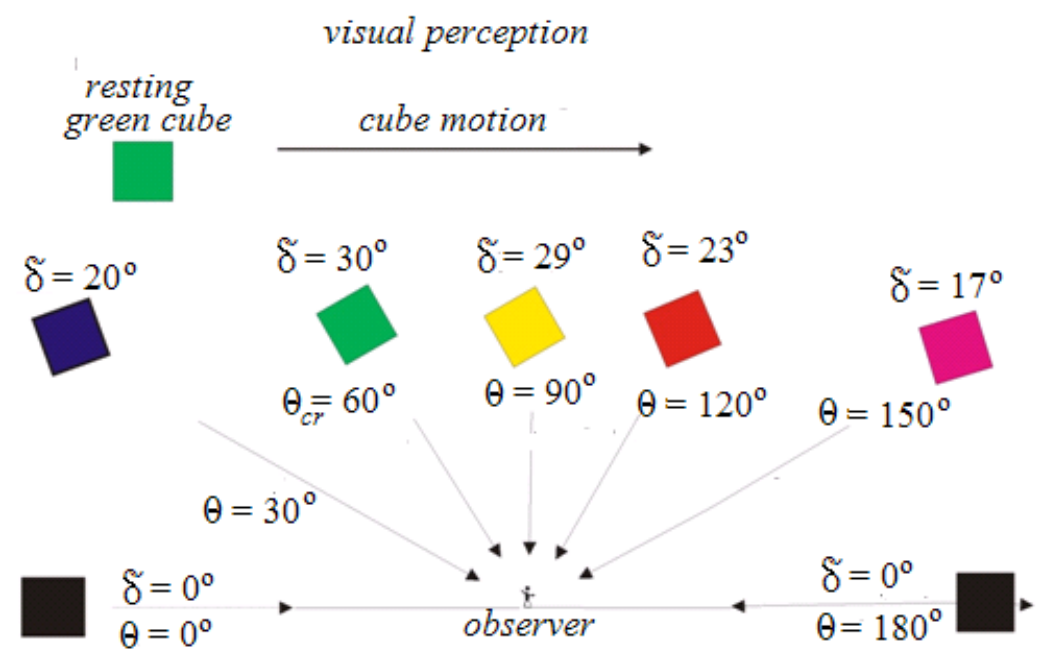

Figure 10: The observed visual orientation of the moving cube in space and the color change of the cube due to the Doppler effect.

We draw attention to the following fact. When moving, the observed object "turns" by the angle of aberration relative to the natural position. This effect resembles the phenomenon of lunar libration.

\section{6. "GEDANKEN EXPERIMENT" BY A. EINSTEIN}

We do not accuse A. Einstein of deliberate mistakes. The level of his philosophical understanding of physical phenomena and the level of understanding of his colleagues were (to put it mildly) not very high. Consider the second part of one of his "mental experiments". Let us turn to [6], where a brief description of the second experiment is given. We quote [6]: 
"The second experiment. Comparison of the clock. When comparing the progress of clocks connected with frames of reference moving relative to each other, it must be remembered that one clock in the system $\Sigma$ can not be compared with one clock in the system $\Sigma^{\prime}$, since the clocks coincide spatially, with each other only at one point in time. ... Let there be a light source at the point where the clock is located in the system $\Sigma^{\prime}$ (Fig. 11 or Fig. 16-2 in [6]). The light signal emitted perpendicular to $\mathbf{v}$ will be reflected by the mirror ... and will return back. For an observer at $\Sigma^{\prime}$, the time necessary for this is equal to $\Delta t^{\prime}=2 z_{0} / c$. An observer resting in $\Sigma$, will measure this time by a couple of hours ... Since the speed of light does not depend on the frame of reference, ...

$$
\Delta t=\Delta t^{\prime} \sqrt{1-v^{2} / c^{2}} \quad(E q .16 .4 \text { in }[6]) .
$$

Note that that the observer in $\Sigma$ will find that his time interval $\Delta t$ is longer than the proper time interval. This phenomenon is known as time dilation."

The comment- This thought experiment can be carried out not only with a mirror. Mirror reinforces the illusion of "correctness" of explanation, intensifies delusion. A mental experiment can be conducted with any moving material body capable of reflecting electromagnetic waves (light). We will use this circumstance.

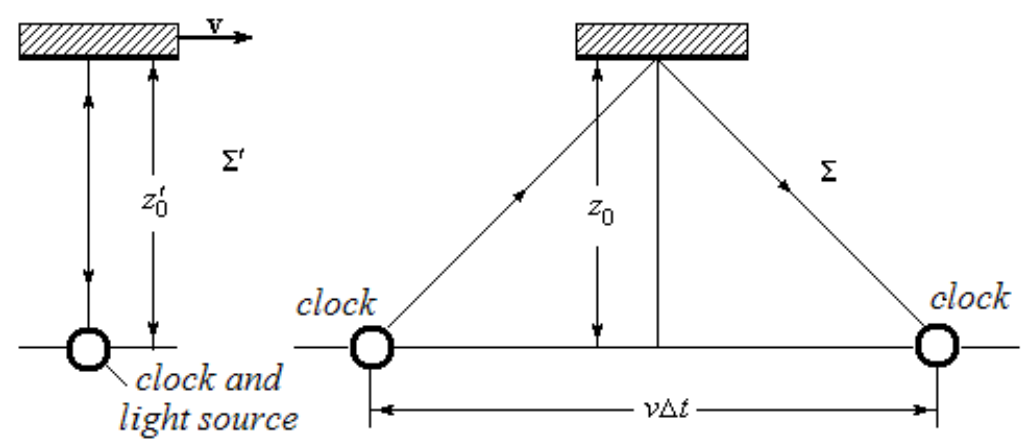

Figure 11: Illustration to Einstein's "thought experiment”

Consider the process in the frame of reference of a stationary observer and break it into two stages:

1) spreading of light from the observer to the moving body and

2) spreading the reflected signal back to the observer.

Consider the process in the frame of reference associated with the observer (Figure 12).

The first stage- At time $t_{1}$, when the moving body passes point 1 , the observer $N$ sends a light signal to point 2 . At time $t_{2}$, the signal occurs at point 2 with the body. Since the light source is stationary, the light ray will travel the distance $R_{01}$ without distortion for observer $N$. This is the actual distance traveled by the light. 


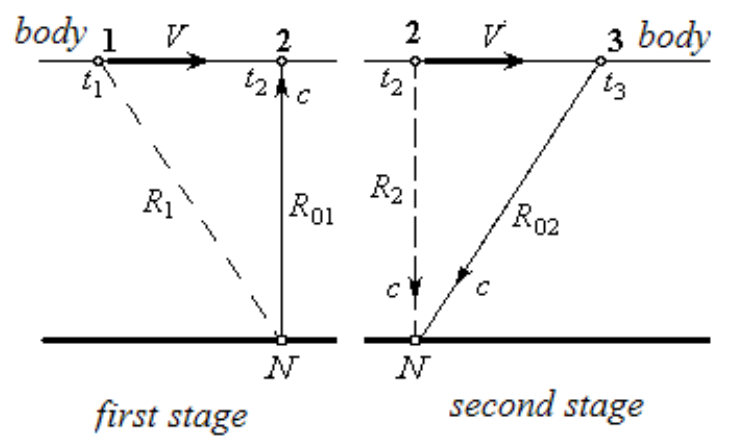

Figure 12: Real distances traveled by the beam in the forward and reverse directions

The second stage- At time $t_{2}$, the light beam is reflected from the body. The observer N, who receives the signal at time $t_{3}$, will appear to have passed the distance $R_{2}$. The observed distance $R_{2}$ is the "apparent" distance (the phenomenon of aberration). At the moment of reception of the reflected light pulse by the observer $N$, the body will be at point 3 . Thus, the actual distance that passed the light in the opposite direction will be equal to $R_{02}$. This seems awesome, but true! So, the distance traveled by the light signal will be equal to the sum of the distances $R_{01}$ and $R_{02}$. Recall that the distances $R_{01}$ and $R_{02}$ are true scalars, that is, they are invariant under the Lorentz transformation. The total time taken to "travel" the signal $T=\left(R_{01}+R_{02}\right) / c$.

Quite analogously, one can consider processes in the frame of reference associated with a moving body and show that the travel time is also equal to $T=\left(R_{01}+R_{02}\right) / c$. Time is one!

We will not consider the "mental experiment" for "proof" of reducing the scale, since in his description the erroneous position of A. Einstein on "slowing down time" is used. There is no real "reduction" in reality. Einstein, expounding the second thought experiment, "forgot" about the existence of the phenomenon of light aberration.

\section{Light and Curvilinear Motion}

\subsection{Curvilinear Motion}

We begin with the general case of curvilinear relative motion. As is known, the Lorentz transformation was derived for the case of rectilinear motion with a constant relative velocity. Is it possible to use this transformation for an arbitrary movement of the object under study? This is an important question.

Suppose that the observer $N$ is at rest in the inertial frame of reference, and the light source $S$ moves along a curvilinear trajectory. The source in position $S^{*}$ emits a light pulse at the time $t_{\mathrm{em}}$. This impulse will be accepted by the observer with some delay at time $t_{\mathrm{ac}}=t_{\mathrm{em}}+R / c$. In Fig. 13 curvilinear segment $S^{*} S$ is the source trajectory in the time interval $R / c$. The straight line segment $S^{*} S^{\prime}$ is a trajectory, provided that the source continues to move linearly with the same constant velocity $\mathbf{V}$. If the source were moving at a rectilinearly constant velocity $\mathbf{V}$, then the Lorentz transformation would predict the true position of the source at the point $S^{\prime}$ at a distance $R^{\prime}$ from the observer, and the aberration angle would be $\delta^{\prime}$, as shown in Figure 13. 


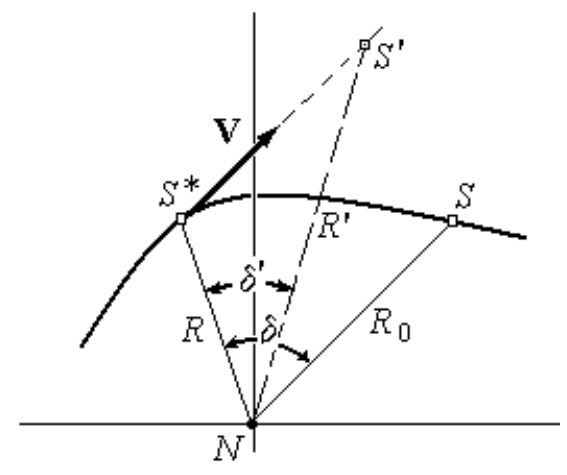

Figure 13: Illustration of curvilinear motion

However, the real trajectory is curvilinear. The actual distance is $R_{0}$, and the aberration angle is $\delta$. These are completely different results. Obviously, we do not have the right to use the Lorentz transformation to describe the motion of an object with variable speed and with curvilinear motion! The theory of accelerators and Ehrenfest's paradox directly testify to this. The misuse of the Lorentz transformation for curvilinear motion leads to erroneous explanations and incorrect mathematical results. For example, when explaining the phenomenon of light aberration in curvilinear motion, there are almost always difficulties.

We will also recall the Ehrenfest paradox. How many hypotheses and "explanations" of a fantastic nature exist in periodicals. However, up to the present moment, we do not have a convincing explanation.

\subsection{Analysis for Rotational Motion}

Critical viewing angle- We return to Chapter 5, in which we met with an amazing critical viewing angle $\Theta_{c r}$. As we found, at a critical viewing angle, the distortion factor is equal to one $\left(n_{d}=1\right)$. Distortions are absent, despite the movement. This is due to the fact that when $\Theta=\Theta_{c r}$. The distance between the observer and the light source remains the same for a short time interval.

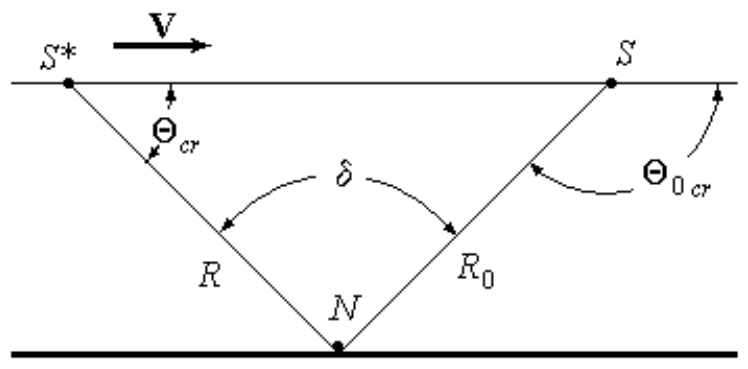

Figure 13

So, at a critical angle of observation we have (Figure 13):

1) The apparent distance $R$ is numerically equal to the actual distance $R_{0}$ between the light source and the observer.

2) There is no Doppler effect. 
3) There are no phenomena of "scale compression" and "time dilation". Therefore, the observed linear velocity will be equal to the actual velocity of motion of the object $V$.

4) The aberration angle at the critical observation angle is maximal.

The angles satisfy the important condition $\Theta_{\mathrm{cr}}+\Theta_{0 \mathrm{cr}}=180^{\circ}$. From the correlation of angles, important relations follow: $\sin \Theta_{\mathrm{cr}}=\sin \Theta_{0 \mathrm{cr}} ; \cos \Theta_{\mathrm{cr}}=-\cos \Theta_{0 \mathrm{cr}}$.

Now we can proceed to an explanation of the phenomena when the light source rotates around the observer.

Inertial reference system- Let us assume that the light source moves around the observer in a circular orbit. The observer is at rest in the center of this orbit. The angular velocity of motion $\Omega$ of the light source is constant. Now we can draw an analogy between the linear motion of the source at a critical observation angle and the motion of the light source posts de circle around the observer $N$. We see general aspects:

When rotating, the distance between the light source and the observer is always constant. Therefore, in the inertial reference frame, the distance distortion coefficient is always $1\left(n_{d}=1\right)$. Due to the constancy of the distance $R$, the Doppler effect is absent. At $n_{d}=1$ there are no phenomena of "compression of scale" and "slowing down of time". Therefore, the observed linear velocity will be equal to the actual velocity of the object's motion $V=\Omega R$. Naturally, the Ehrenfest paradox is absent. The angles satisfy the important condition $\Theta_{\mathrm{cr}}=\Theta_{0 \mathrm{cr}}=90^{\circ}$. From the correlation of angles, important relations follow: $\sin \Theta_{\mathrm{cr}}=\sin \Theta_{0 \mathrm{cr}}=1 ; \cos \Theta_{\mathrm{cr}}=-\cos \Theta_{0 \mathrm{cr}}=$ 0 .

We see here a complete analogy between phenomena at a critical viewing angle for linear motion and for rotational motion.

Angle of aberration. Let the light source $S^{*}$ emit a light pulse at the time $t=t_{\mathrm{em}}$. The observer $\mathrm{N}$ will accept this pulse at time $t=t_{\mathrm{ac}}$. For a time equal $t_{\mathrm{em}}-t_{\mathrm{ac}}=T=R / c$, the moving source will have time to occupy the position $S$. (See Figure 14).

The length of the $\operatorname{arc} S S^{*}$ is

$$
S S^{*}=\Omega R T=\Omega R^{2} / c
$$

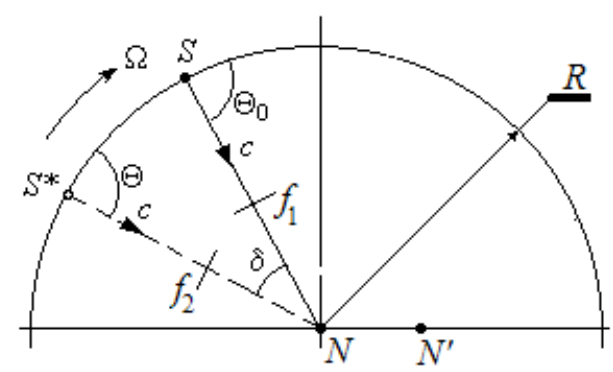

Figure 14: Inertial reference system. $f_{1}$ is wave front from the source in its reference frame; $f_{2}$ is distorted wave front, perceived by the observer. 
The angle of aberration is: $\delta=S S^{*} / R=\Omega R / c$. This is true if the observer is at the point $N$. If he moves to the point $N^{\prime}$ (Fig. 14), then immediately there will be changes:

- The distortion ratio $\mathrm{nm}$ will periodically change over time.

- There will be a phenomenon of libration.

- The observed linear and angular velocity will have a deviation.

- The Doppler effect appears, etc.

- Similar phenomena occur in a non-inertial rotating frame of reference.

Noninertial reference system- We write the wave equation in a cylindrical coordinate system.

$$
\frac{1}{r} \frac{\partial}{\partial r}\left(r \frac{\partial u}{\partial r}\right)+\frac{1}{r^{2}} \frac{\partial u^{2}}{\partial \varphi^{2}}+\frac{\partial u^{2}}{\partial z^{2}}-\frac{1}{c^{2}} \frac{\partial u^{2}}{\partial t^{2}}=0 .
$$

It turns out that for equation (7.2.2) there exists a transformation analogous to the Lorentz transformation for a fixed distance $r=R_{0}$ :

$$
\begin{gathered}
r=R_{0} ; \quad \varphi_{0}=\varphi \sqrt{1+\left(\Omega_{0} R_{0} / c\right)^{2}}-\Omega_{0} t ; \\
z_{0}=z ; \quad c t_{0}=c t \sqrt{1+\left(\Omega_{0} R_{0} / c\right)^{2}}-R_{0}^{2} \Omega_{0} / c ;
\end{gathered}
$$

$\varphi_{0}=\varphi=0$ for $t=t_{0}=0$

This transformation preserves the form of the wave equation in the rotating frame of reference. Here, instead of the velocity $V$, the angular velocity of rotation $\Omega_{0}$ appears. For the analysis, we make the following substitutions:

- $\quad$ the product $\Omega_{0} R_{0}$ is replaced by $V\left(\Omega_{0} R_{0}=V\right)$;

- rotation angles are replaced by arcs: $s_{0}=R_{0} \varphi_{0} ; s=R_{0} \varphi$.

Then for $r=R_{0}$, the transformation (7.2.3) takes the form similar to the standard Lorentz transformation.

$$
\begin{gathered}
s_{0}=s \sqrt{1+V^{2} / c^{2}}-V t ; r=R_{0} \\
z_{0}=z ; \quad c t_{0}=c t \sqrt{1+V^{2} / c^{2}}-s V / c .
\end{gathered}
$$

Obviously, this transformation is valid only for the radius $r=R_{0}$. There is no movement on the circle of this radius. For other radii, virtual rotation takes place, as shown in Figure 15.

- If $r$ is greater than $R_{0}$ then the direction of rotation is clockwise, as shown in Fig. 15.

- If $r$ is equal to $R_{0}$, we see a fixed circle.

- If $r$ is smaller than $R_{0}$ then the direction of rotation is counterclockwise.

- The observer $\mathrm{N}$ in the center rotates at a speed of $\Omega_{0}$ counterclockwise.

This task has a cumbersome solution. The solution of the problem has a simple form for small velocities $(V \ll c)$. The transformation for this case is simplified: 


$$
\begin{gathered}
s_{0} \approx s-V t ; \quad R_{0}=\mathrm{const} ; \\
z_{0}=z=0 ; \quad c t_{0}=c t-s V / c \approx c t .
\end{gathered}
$$

On a stationary circle of radius $\mathrm{R} 0$, the source $\mathrm{S}$ is at rest (Fig. 15). Let the source emit a light pulse to the observer. The trajectory of the light pulse in the previously considered inertial frame of reference is as follows

$$
r=R_{0}-c t=R_{0}-c t_{0}
$$

where $r$ is the distance traveled by the light pulse from $S$ from the moment of radiation.

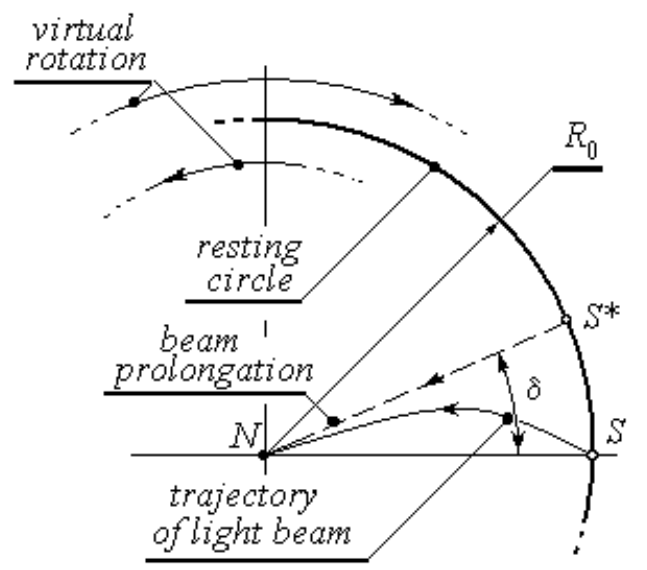

Figure 15: Light beam in a rotating frame of reference

Eliminate the time from the first equation (7.2.5) using (7.2.6), in addition to the expression (7.2.6), we obtain the second equation for describing the trajectory in the noninertial reference frame:

$$
\varphi_{0}=s_{0}\left(t_{0}\right) / R_{0}=\Omega_{0}\left(R_{0}-r\right)^{2} / R_{0} c .
$$

As expected, the ray trajectory has a curvilinear character (see Figure 15). The aberration angle can be found under the following condition: $t=R_{0} / c$ or $r=0$. It is equal to

$$
\delta=\Omega_{0} R_{0} / c .
$$

This result corresponds to the result obtained above for an inertial frame of reference.

Note- Let the observer rest in the inertial frame of reference. He observes how a charged particle moves with a variable velocity along a curved trajectory. The observer measures the fields $\mathbf{E}$ and $\mathbf{H}$ of the moving charge. Can we calculate these fields at the place where the observer is resting, using a modified Lorentz transformation or transformation? Modern physics gives a positive answer. However, this is the wrong answer. We have seen that rectilinear motion and rotational motion give different effects. We shall show this below in the example of cyclic accelerators.

The above analysis leads us to the following conclusions:

- In the general case, the Lorentz transformation does not apply to curvilinear motion. 
- The trajectory of a light beam in a non-inertial frame of reference can be curvilinear.

- "Compression" of the circle and "slowing down" of time are absent. However, the length of the trajectory of the light beam is greater than the radius. Therefore, the ratio of the length of the circle to the length of the light beam is less than $2 \pi$. We have a logically correct explanation of Ehrenfest's paradox.

\subsection{Accelerators}

It is believed that the work of cyclic accelerators of elementary particles serves as a reliable experimental confirmation of the special theory of relativity. It's easy to check. The conclusions obtained earlier are directly related to the theory of cyclic accelerators. We consider a special case of motion with a constant velocity along a circular orbit. But first we will give an explanation. Suppose hypothetically that an electron moving rectilinearly and uniformly falls into a homogeneous magnetic field. Obviously, in this field the trajectory will be a circle.

For a relativist, the velocity of a particle in a circular motion in a magnetic field will be the same as for a rectilinear motion before entering the magnetic field, i.e. $v_{0}$ (the velocity entering into the Lorentz transformation).

The materialist will reason otherwise. A rectilinearly moving electron has a real velocity $V$. At the same time, the velocity observed with the help of light rays with rectilinear motion will be $v_{0}$. The connection between them is simple $V=v_{0} / \sqrt{1-v_{0}^{2} / c^{2}}$. When the transition from the translational phase of the motion to the rotational momentum of an electron and its real velocity $V$ do not change. This we saw on the example of the explanation of the Ehrenfest paradox. At the same time, the observed velocity will be different for these phases of motion. In the transition from translational motion to rotational motion, the value of the observed velocity jumps by the factor $1 / \sqrt{1-v_{0}^{2} / c^{2}}$.

In essence, the use of this or that speed is related to what we want to describe: the motion of the source (instantaneous mapping) or the movement of its imaginary mapping transmitted by light rays. The theory of relativity of A. Einstein is focused only on the description of the imaginary image. But she mistakenly considers an imaginary image as an actual material object. Let us see what results follow from its provisions for cyclic accelerators.

Let a charged particle with velocity $v_{0}$ enter a uniform magnetic field perpendicular to its lines of force. It is known from SRT that the velocity of a particle can not exceed the speed of light in a vacuum (Einstein's postulate). Thus, in SRT particles can have a velocity $v_{\mathrm{obs}}=0.99 c, v_{\mathrm{obs}}=$ $0.999 c$ or $v_{\text {obs }}=0.9999 c$, and so on. The angular velocity of rotation of particles at such velocities should be practically the same according to SRT. It is approximately equal to $c / R$. In fact, this is not so!

Let us consider a concrete example. The ARCS synchrotron has the following technical characteristics: [7]:

- length of the orbit is $2 \pi R=216.7 \mathrm{~m}$;

- energy of electron injection $\mathrm{W}=50 \mathrm{MeV}$; 
- frequency of the accelerating field $f=132.8 \mathrm{MHz}$;

- the acceleration multiplicity $g=96$;

- rest energy of the electron $E_{0}=0.511 \mathrm{MeV}$.

According to the formula following from the special theory of relativity, the frequency of electron bunches in the orbit of the ARCS accelerator at the moment of electron injection at the kinetic energy of electrons $W=48.55 \mathrm{MeV}$ will be equal to

$$
f_{\mathrm{SRT}}=\frac{c \sqrt{\left(\frac{W}{E}+1\right)^{2}-1}}{2 \pi R\left(\frac{W}{E}+1\right)}=1.3843 \mathrm{MHz} .
$$

The actual frequency of the accelerating field in the accelerator is much higher. It is equal to $f=$ 132.8 MHz. The period $T=1 / f=7.53 \mathrm{~ns}$ of revolution of electron bunches along an orbit of length $216.7 \mathrm{~m}$ would mean that electrons move with a velocity 96 times faster than the speed of light. According to the special theory of relativity, superluminal electron velocities are impossible. Therefore, in order to explain the experimental value of the target irradiation period $7.53 \mathrm{~ns}$ within the framework of the special theory of relativity, it was required to introduce the concept of "acceleration multiplicity" $g$ and to declare that "under the action of the accelerating field, the particles of the injected beam decay into bunches clustered around stable equilibrium phases. The number of such clusters, which are located along the circumference of the accelerator, is equal to the acceleration multiplicity $g$ " [7]. Indeed, dividing the value of $f=132.8 \mathrm{MHz}$ by the value $f_{\text {SRT }}$ $=1.384 \mathrm{MHz}$, we obtain $g=96$. This is the multiplicity of the acceleration of the electron synchrotron ARUS. We note that the multiplicity of the acceleration of the proton synchrotron CERN in the experiment [8], for example, is 19.

Thus, the experimental values of the frequency of revolution of the clusters of elementary particles in the two accelerators considered do not confirm the formula (7.3.1) from the special theory of relativity. To explain the experimental values of the frequency of revolution of elementary particle bundles within the framework of the special theory of relativity and the agreement of these values with formula (7.3.1), a special hypothesis is used, based on the introduction of ad hoc of the concept of "acceleration multiplicity" $g$. In some textbooks on the theory of particle accelerators, the hypothesis is called "witty." Supporters of the STR were unable to understand the cause of this phenomenon. When theory and experiment "run up," dogmatists usually use three techniques:

1) Keep silent about the experiment.

2) "Adjust" the experimental results under the predictions of the theory.

3) Introduce a questionable "postulate" or ad hoc hypothesis to "connect" the theory with practice.

Now even theoreticians had to introduce the ad hoc hypothesis about the existence of the acceleration multiplicity $g$. In fact, there is no "decay into clusters grouped around stable equilibrium phases" in a synchrotron. This is a fantasy, an invention. To do this, it is sufficient to consider a single electron that enters the accelerator. Is this single electron also "broken up into clumps clustered around stable equilibrium phases"? This is obvious nonsense! 
We have already explained above that the actual velocity of particles $V$ is greater than their observed velocity $v_{0}$, which is part of the Lorentz transformation. It is equal to $V=$ $v_{0} / \sqrt{1-v_{0}^{2} / c^{2}}$. It is with such a linear velocity (in spite of the prohibitions of SRT) that charged particles move around the circumference in the accelerator considered above. To estimate, we calculate this speed. Let the observed velocity with linear motion of the charge electron be equal to $v_{0}=0.99995 \mathrm{c}(\approx 50 \mathrm{MeV})$. Then the value of the actual velocity of the charged particle will be $V \sim 100 c$.

Correction of the epistemological error in the theory of cyclic accelerators is not a simple replacement of the observed velocity $v_{0}$ at an angle of $90^{\circ}$ in the Lorentz transformation by the real velocity $V$. There is a need for a revision of relativistic mechanics. In addition, the previously obtained results of experimental measurements on accelerators will need to be rethought, etc.

\section{Note}

1) In the theory of relativity, the Lorentz transformation was used to map the fields $\mathbf{E}$ and $\mathbf{H}$ of the moving charge to the inertial reference frame of the observer for any (even curvilinear) motion. The use of a modified transformation or the Lorentz transformation in non-inertial frames of reference is generally unacceptable. The mapping of fields in the curvilinear motion of a particle is a special topic.

2) Within the framework of Maxwell's equations, as we have said, there exist instantaneous fields of inertial charges. This problem also requires an independent presentation. We note that the principle of causality with instantaneous action at a distance is not violated.

\section{Instead of Conclusion}

The purpose of our work was to analyze the reasons that divided the scientific community into two warring camps. It turned out that the cause of the contradictions was the misunderstanding and use by the scientific community of the philosophical categories "phenomenon and essence". This situation developed historically by the end of the 19th century. There are no guilty persons here, but there is a common misconception. A common error led to dogmatism, which hindered the development of new ideas.

We will not list the results we obtained. We sought to fully describe the physical meaning of the phenomena associated with light and inertial frames of reference. Here we draw the reader's attention to the philosophy of physics and its state.

Modern positivism defines the role of philosophy as a methodology of science. But even the most advanced methods in science are powerless if the scientist does not have criteria or attributes that allow him to separate the truth from errors and errors. The elaboration and comprehension of a system of such attributes (criteria) of general scientific and private character is an urgent problem of the philosophy of science in general and of physics in particular. Without solving this problem, physics will long be chosen from the crisis that arose in the beginning of the 20th century and continues at the present time. 
We used two attributes (or criteria) in our analysis:

- The principle of logical consistency of the content of scientific theories.

- Correct understanding of the difference between philosophical categories "phenomenon and essence" ("golden rule").

It is now difficult to assess the consequences, which will lead to a new interpretation of the Lorentz transformation. At least, specialists in the field of theoretical and technical physics will have to revise the theory of the interaction of elementary particles. Experimenters also have a lot of technical work to do. They must reconsider and recount the results of numerous experiments already conducted, etc. Revision of theories and experimental results is the price of an epistemological error. Note that this is not the only error that arose in the crisis of physics. For example, we found that there are reasons for revising certain positions in modern electrodynamics [9].

The physical interpretation of phenomena is a reflection of the worldview (philosophical views) of the scientist. Physicists and philosophers together must develop the philosophy of natural science. It is appropriate here to recall the words of the materialist philosopher F. Engels [10] that materialistic philosophy is like a capricious lady. She avenges natural science in retrospect for the fact that the latter left her.

\section{Appendices}

\section{Appendix A. Class of Transformations}

As is known, Poincaré in 1904 put forward a provision according to which in all, without exception, inertial systems, the laws of nature are the same [11]. The speed of light is a quantity that is invariant, independent of the choice of the frame of reference by the observer.

In Chapter 2 we got acquainted with the philosophical categories "phenomenon and essence". The observer and the object under investigation are bound by the information carrier, which transmits information from the observation object to the observer. In SRT such a medium of information is light rays. As we now know, the essence does not depend on the conditions of observation of phenomena, i.e. from the information carrier. In SRT, true scalars are invariants that are independent of the relative velocity of inertial systems. True scalars are characteristics of aspects of an essence.

The question arises. Is the Lorentz transformation applicable to all phenomena without exception, or is it applicable only to an electromagnetic wave? This problem requires special study. So, let us look for a class of 4-coordinate transformations for which the wave equations retain their shape in accordance with the Galileo-Poincaré principle.

Let us consider two inertial reference frames $K$ and $K_{0}$, which move relative to each other with velocity $\mathrm{V}$ along the $\mathrm{x}$ axis. The space-time coordinates of the system $K(x \cdot y \cdot z, c t)$ must be related to the corresponding coordinates $K_{0}\left(x_{0}, y_{0}, z_{0}, c t_{0}\right)$ using the transformation matrix $[\boldsymbol{T}(V / c)]$.

$$
\left[\boldsymbol{X}_{0}\right]=[\boldsymbol{T}(V / c)][\boldsymbol{X}]
$$


where $[\boldsymbol{X}]$ and $\left[\boldsymbol{X}_{0}\right]$ are the vector columns of the 4-coordinates $K$ and $K_{0},[\boldsymbol{T}(V / c)]$ is the transformation matrix, which depends only on the relative motion speed of the compared inertial systems.

The following requirements are imposed on the matrix $[\boldsymbol{T}]$ :

- the determinant of the matrix must be equal to one: $\operatorname{det}[\boldsymbol{T}]=1$;

- there must exist an inverse transformation matrix from $K_{0}$ to $K$, i.e. the matrix $[\boldsymbol{T}(V / c)]^{-1}$;

- the inverse transformation matrix must be obtained by replacing $V$ by $-V$. This follows from the equality of inertial reference systems $[\boldsymbol{T}(V / c)]^{-1}=[\boldsymbol{T}(-V / c)]=[\boldsymbol{E}]$. Composition; where $[\boldsymbol{E}]$ is the unit diagonal matrix.

From these conditions it is possible to determine the general form of the matrix of transformations of coordinates and time preserving the invariant form of the wave equations. Equations corresponding to (A1.1) can be written in the following form:

$$
\begin{gathered}
x_{0}=x \sqrt{1+f^{2}(V / c)}-c t f(V / c) ; y_{0}=y ; z_{0}=z ; \\
c t_{0}=c t \sqrt{1+f^{2}(V / c)}-x f(V / c)
\end{gathered}
$$

where $f(V / c)$ is an odd function with respect to $V / c$. The conditions listed above are not sufficient, unfortunately, to determine the explicit form of the function $f(V / c)$. It can be $V / c$, or $\sin (V / c)$, or $\sinh (V / c)$, etc.

At low velocities $f(V / c) \approx V / c$ :

$$
x_{0}=x\left[1+(V / c)^{2} / 2\right]-V t ; y_{0}=y ; z_{0}=z ; c t_{0}=c t\left[1+(V / c)^{2} / 2\right]-x(V / c) .
$$

Special cases:

- If $f(V / c)=V / \sqrt{c^{2}-V^{2}}$ then we have the Lorentz transformation

$x_{0}=(x-V t) / \sqrt{1-(V / c)^{2}} ; y_{0}=y ; z_{0}=z ; c t_{0}=(c t-x V / c) / \sqrt{1-(V / c)^{2}}$.

- If $f(V / c)=V / c$ then we have a modified transformation

$x_{0}=x \sqrt{1+(V / c)^{2}}-V t ; y_{0}=y ; z_{0}=z ; c t_{0}=c t \sqrt{1+(V / c)^{2}}-x V / c$.

- If $f(V / c)=\sinh (V / c)$ then we have a hyperbolic transformation

$$
x_{0}=x \cosh (V / c)-c t \sinh (V / c) ; y_{0}=y ; z_{0}=z ; c t_{0}=c t \cosh (V / c)-x \sinh (V / c)
$$

We believe that direct measurements of the velocity of charges are necessary in the presence of the necessary magnitude of the accelerating potential $U_{0} \geq m c^{2} / e$. Modern experimental techniques make it possible to implement such an experiment. 


\section{Appendix B. Group Properties}

For each function $f(V / c)$ in expression (A.1.2) there corresponds a transformation that forms a group. Such a group is non-commutative. This property creates insurmountable difficulties in describing phenomena. We illustrate it with elementary examples.

Let the light source $S$ move along the x axis with the velocity $\mathbf{V}$, as shown in Fig. 16. We need to find a reference frame where this source is at rest.

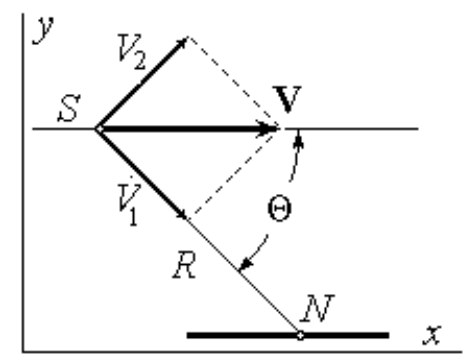

Figure 16

Observer $N$ sees this source at an angle $\Theta$. We can go to the desired frame in several ways. For example, we can decompose the vector $\mathbf{V}$ into a sum of two orthogonal components. One component $V_{1}=V \cos \Theta$ is directed towards the observer $N$, the other component $V_{2}=V \sin \Theta$ has an orthogonal direction. We can, for example, use the transformation $[\boldsymbol{T}(V \cos \Theta / c)]$ first, and then apply the transformation $[\boldsymbol{T}(V \sin \Theta / c)]$. The general transformation has the form:

$$
[\boldsymbol{T}(V / c)]=[\boldsymbol{T}(V \cos \Theta / c)] \cdot[\boldsymbol{T}(V \sin \Theta / c)]
$$

But we can swap the transformation matrices:

$$
[\widetilde{\boldsymbol{T}}(V / c)]=[\boldsymbol{T}(V \sin \Theta / c)] \cdot[\boldsymbol{T}(V \cos \Theta / c)]
$$

Using the expression (A.2.1) or the expression (A.2.2), we find an inertial system in which the light source $S$ is at rest:

$$
\left[\boldsymbol{X}_{0}\right]=[\boldsymbol{T}(V / c)] \cdot[\boldsymbol{X}] \text { and }\left[\widetilde{\boldsymbol{X}_{0}}\right]=[\widetilde{\boldsymbol{T}}(V / c)] \cdot[\boldsymbol{X}]
$$

where $[\boldsymbol{X}]$ there is a 4-matrix-column $(x, y, z, c t)$. Obviously, we will get two different results, i.e. we find two different reference frames:

$$
\left[\boldsymbol{X}_{0}\right] \neq\left[\widetilde{\boldsymbol{X}_{0}}\right]
$$

This is the first difficulty caused by the non-commutativity of the group.

Let us now consider the second difficulty. We will try, with the help of the inverse transformation, to return the particle back to our frame of reference. It is possible to quickly find the matrix of the 
inverse transformation if in the matrix of direct transformation we replace the sign of the velocity $V$ by the opposite:

$$
[\boldsymbol{T}(V / c)]^{-1}=[\boldsymbol{T}(-V / c)]
$$

However, because of non-commutativity, we cannot return the source to our frame of reference to the same point.

$$
\begin{gathered}
{[\boldsymbol{T}(-V / c)] \times[\boldsymbol{T}(V / c)]^{-1}=[\boldsymbol{T}(V / c)] \times[\boldsymbol{T}(-V / c)]} \\
=[\boldsymbol{T}(V \cos \Theta / c)] \cdot[\boldsymbol{T}(V \sin \Theta / c)][\boldsymbol{T}(-V \cos \Theta / c)] \cdot[\boldsymbol{T}(-V \sin \Theta / c)] \\
\neq[\boldsymbol{E}]
\end{gathered}
$$

where $[\boldsymbol{E}]$ there is a unit matrix.

Now we can make a hypothesis and answer the question: "Why did not Poincaré defend his priority in creating SRT?" Poincare saw that the Lorentz group is not a commutative group. He realized that the direction he had proposed had no prospects. Poincaré lost interest in this idea and did not defend his priority. Perhaps he was looking for a new solution, but premature death violated plans...

\section{References}

[1] Crother S. (2017). "ON THE LOGICAL INCONSISTENCY OF THE SPECIAL THEORY OF RELATIVITY.” American Journal of Modern Physics, 6, 43-48.

[2] Leus V. (2018). "CRITICAL COMMENTS ON THE PAPER 'ON THE LOGICAL INCONSISTENCY OF THE SPECIAL THEORY OF RELATIVITY'.” Journal of Applied Mathematics and Physics, 6 925-931.

[3] Poincaré H. (1906). “SUR LA DYNAMIQUE DE L’ÉLECTRON.” Ren. Circ. Mat. Palermo, 21, 129-175.

[4] Lenin V. I. (2002). Materialism and Empirio-Criticism: Critical Comments on a Reactionary Philosophy (Univ. Press of the Pacific).

[5] Kuligin V., Kuligina G., and Korneva M. (1994). "EPISTEMOLOGY ANDSPECIAL RELATIVITY.” Apeiron, 20, 21.

[6] Panofsky W. and Phillips M. (1990). Classical Electricity and Magnetism (2nd ed., Dover Publ.).

[7] Burshtein E. (2011). "ACCELERATORS OF CHARGED PARTICLES" Great Soviet Encyclopedia (Translation from Russian into English University Library of Nijmegen).

[8] Alvager T., Farley F., Kjellman J., and Wallin J. (1964). "TEST OF THE SECOND POSTULATE OF SPECIAL RELATIVITY IN THE GEV REGION.” Physical Letters, 12(3), 260 -262.

[9] Chubykalo A., and Kuligin V. (2018). "UNKNOWN CLASSICAL ELECTRODYNAMICS." Boson Journal of Modern Physics, 4(2), 384-425.

[10] Engels F. (1964). Dialectics of Nature (3rd ed. Progress Publishers).

[11] Martínez A. (2004). "RITZ, EINSTEIN, AND THE EMISSION HYPOTHESIS." Physics in Perspective, 6(1), 4-28.

*Corresponding author.

E-mail address: achubykalo@ yahoo.com.mx 